\title{
WEAKLY PERIODIC AND SUBWEAKLY PERIODIC RINGS
}

\author{
AMBER ROSIN and ADIL YAQUB
}

Received 30 October 2002

\begin{abstract}
Our objective is to study the structure of subweakly periodic rings with a particular emphasis on conditions which imply that such rings are commutative or have a nil commutator ideal. Related results are also established for weakly periodic (as well as periodic) rings.
\end{abstract}

2000 Mathematics Subject Classification: 16U80, 16D70.

Throughout, $R$ represents a ring and $\mathscr{C}(R)$ denotes the commutator ideal of $R$. For any $x, y$ in $R,[x, y]=x y-y x$ is the usual commutator. A word $w(x, y)$ is a product in which each factor is $x$ or $y$. The empty word is defined to be 1 . We now state formally the definition of a subweakly periodic ring.

DEFINITION 1. A ring $R$ is called subweakly periodic if every $x$ in $R \backslash J$ can be written in the form $x=a+b$, where $a$ is nilpotent and $b$ is potent.

In the preparation for the proofs of the main theorems, we first prove the following lemmas.

LEMMA 2. Suppose that $R$ is any ring with the property that for all $x, y$ in $R$, there exist words $w(x, y), w^{\prime}(x, y)$ depending on $x$ and $y$ such that

$$
w(x, y)[x y, y x]=0=[x y, y x] w^{\prime}(x, y) \quad(x, y \in R) .
$$

Then the commutator ideal $\mathscr{C}(R)$ is contained in $J$. In particular, if $R$ is also semisimple, then $R$ is commutative.

Proof. The semisimple ring $R / J$ is isomorphic to a subdirect sum of primitive rings $R_{i}, i \in \Gamma$, each of which clearly satisfies (1).

CASE 1. Suppose that $R_{i}$ is a division ring. We claim that $R_{i}$ must be commutative. Suppose not. Let $x, y \in R_{i}$ be such that $[x, y] \neq 0$. As $x \neq 0$ and $y \neq 0$, we must have $w(x, y) \neq 0$, and so by (1), $[x y, y x]=0$. Since $[x, y] \neq 0$ implies that $[x, y+1] \neq 0$, we may repeat the above argument, with $y+1$ playing the role of $y$, to conclude that $[x(y+1),(y+1) x]=0$, and hence $[x y+x, y x+$ $x]=0$. Combining this with $[x y, y x]=0$, we obtain $[x y, x]+[x, y x]=0$, and thus $[x y-y x, x]=0$, that is, $[x, y]$ commutes with $x$. Interchanging $x$ and $y$ in the above argument, we see that $[y, x]$ commutes with $y$, and hence

$$
[x, y] \text { commutes with both } x \text { and } y \text {. }
$$


Observe that $[x y, x]=x[y, x]$, and hence

$$
x=[x y, x][y, x]^{-1} .
$$

By (2), $[x y, x]$ commutes with both $x$ and $x y$, and hence $[x y, x]$ commutes with $x^{-1}(x y)=y$. Again, by (2), $[y, x]$ commutes with $y$, and hence $[y, x]^{-1}$ also commutes with $y$. The net result is that both factors of the right-hand side of (3) commute with $y$, and hence $[x, y]=0$, contradiction. This contradiction proves that the division ring $R_{i}$ is commutative.

CASE 2. The primitive ring $R_{i}$ is not a division ring. Since (1) is inherited by all subrings and all homomorphic images of the ground ring $R$, it follows, by Jacobson's density theorem [4, page 33], that for some $n>1$ and some division ring $D$, the complete matrix ring $D_{n}$ of all $n \times n$ matrices over $D$ satisfies (1). This, however, is false as can be seen by taking $x=E_{11}, y=E_{11}+E_{12}$ $\left(x, y \in D_{n}\right)$. Indeed, in this case, any word $w(x, y)$ must be $x$ or $y$ (since $x^{2}=x, y^{2}=y, x y=y$, and $\left.y x=x\right)$, which implies that

$$
w(x, y)[x y, y x]=w(x, y)[y, x]=x[y, x]=x-y \neq 0
$$

or

$$
w(x, y)[x y, y x]=w(x, y)[y, x]=y[y, x]=x-y \neq 0 .
$$

This contradiction shows that each $R_{i}$ must be a division ring, and hence must be commutative (by Case 1). Therefore, $R / J$ is commutative, and hence $\mathscr{b}(R) \subseteq$ $J$. This proves Lemma 2.

LEMмA 3. Suppose that $R$ is a ring which satisfies the "word" hypothesis (1) of Lemma 2. Suppose, further, that $J$ is commutative. Then the commutator ideal of $R$ is nil, and hence $N$ is an ideal of $R$.

Proof. By Lemma 2, every commutator $[x, y]$ is in $J$. Since, by hypothesis, $J$ is commutative,

$$
[[x, y],[z, w]]=0 \quad \forall x, y, z, w \in R
$$

Observe that (6) represents a polynomial identity which is satisfied by all the elements of the ground ring $R$. Moreover, the greatest common divisor of all the coefficients of this polynomial is 1 . Furthermore, (6) is not satisfied by any $2 \times 2$ matrix ring over $\mathrm{GF}(p)$ for any prime $p$, as a consideration of the following commutators shows:

$$
[x, y]=\left[E_{11}, E_{12}\right], \quad[z, w]=\left[E_{22}, E_{21}\right] .
$$

It follows from [1] that the commutator ideal $\mathscr{C}(R)$ is nil, and hence $N$ is an ideal of $R$. This proves Lemma 3. 
LEMmA 4. Suppose that $R$ is a subweakly periodic ring which satisfies all the hypotheses of Lemma 3. Then for any $x$ in $R$,

$$
x \in J \quad \text { or } \quad x-x^{n} \in N \quad \text { for some integer } n>1 .
$$

Proof. Let $x \in R, x \notin J$. Then, by Definition 1 ,

$$
x=a+b, \quad a \in N, b^{n}=b, \text { where } n=n(b)>1 .
$$

Now, by Lemma 3, $N$ is an ideal of $R$, and since $a \in N$,

$$
x^{n}=(a+b)^{n}=a_{0}+b^{n}, \quad a_{0} \in N .
$$

Thus,

$$
x-x^{n}=a+b-a_{0}-b^{n}=a-a_{0} \quad\left(\text { since } b^{n}=b\right),
$$

and hence $x-x^{n} \in N$. This proves Lemma 4 .

LEMMA 5. Suppose that $R$ is a subweakly periodic ring which satisfies all the hypotheses of Lemma 4. Suppose that $\sigma: R \rightarrow S$ is a homomorphism of $R$ onto a ring $S$. Then the set $N^{\prime}$ of nilpotents of $S$ is contained in $\sigma(J)$, and hence $N^{\prime}$ is a commutative set.

Proof. Suppose that $s \in N^{\prime}$ with $s^{k}=0$. Let $d \in R$ such that $\sigma(d)=s$. If $d \in J$, then $s=\sigma(d) \in \sigma(J)$, and the lemma follows. So, suppose that $d \notin J$. Then by Lemma 4,

$$
d-d^{n} \in N \text { for some integer } n>1 \text {. }
$$

It is readily verified that

$$
d-d^{k+1} d^{k(n-2)}=\left(d-d^{n}\right)+d^{n-1}\left(d-d^{n}\right)+\cdots+\left(d^{n-1}\right)^{k-1}\left(d-d^{n}\right) .
$$

Combining (12), (13), and the fact that $N$ is an ideal of $R$, we see that

$$
d-d^{k+1} d^{k(n-2)} \in N
$$

and hence

$$
s-s^{k+1} s^{k(n-2)}=\sigma\left(d-d^{k+1} d^{k(n-2)}\right) \in \sigma(N) .
$$

This implies (since $s^{k}=0$ ) that $s \in \sigma(N)$. Thus, $N^{\prime} \subseteq \sigma(N)$. By Lemma 3, $N$ is an ideal of $R$, and hence $N \subseteq J$, which implies $N^{\prime} \subseteq \sigma(N) \subseteq \sigma(J)$. Finally, since $J$ is commutative, $\sigma(J)$ is commutative, and thus $N^{\prime}$ is a commutative set. 
LEMma 6. Suppose that $R$ is any ring which satisfies the "word" hypothesis (1) of Lemma 2. Then the set $E$ of all idempotents of $R$ is contained in the center of $R$.

Proof. Suppose that $e^{2}=e \in R, x \in R$, and $f=e+e x-e x e$. Then

$$
f^{2}=f, \quad e f=f, \quad f e=e, \quad[e, f]=e x-e x e .
$$

By the "word" hypothesis (1) of Lemma 2, there exists a word $w(e, f)$ such that

$$
w(e, f)[e f, f e]=0 .
$$

By (16), we see that $w(e, f)=e$ or $w(e, f)=f$, and hence (17) is equivalent to

$$
e[e f, f e]=0 \quad \text { or } \quad f[e f, f e]=0 .
$$

These two equations, in turn, are equivalent to $e-f=0$, and hence

$$
e x=e x e
$$

Now, let $f^{\prime}=e+x e-e x e$. Again, by the second part of the "word" hypothesis (1) of Lemma 2, there exists a word $w^{\prime}\left(e, f^{\prime}\right)$ such that

$$
\left[e f^{\prime}, f^{\prime} e\right] w^{\prime}\left(e, f^{\prime}\right)=0
$$

An argument similar to the one above shows that (20) is equivalent to $e-f^{\prime}=0$, and hence

$$
x e=e x e
$$

Combining (19) and (21), we obtain the lemma.

LEMMA 7. Suppose that $R$ is a subweakly periodic ring which satisfies all the hypotheses of Lemma 4. Then, for any $x$ in $R, x$ is in $J$ or $x^{q}=x^{q}$ e for some idempotent $e$ and some $q \geq 1$.

Proof. Let $x \in R$. By Lemma 4,

$$
x \in J \quad \text { or } \quad x-x^{n} \in N \text { for some integer } n>1 \text {. }
$$

Suppose that $x \notin J$. Then, $\left(x-x^{n}\right)^{q}=0$ for some positive integer $q$, and hence

$$
x^{q}=x^{q+1} g(x) \text { for some polynomial } g(\lambda) \in \mathbb{Z}[\lambda] \text {. }
$$

By reiterating, we see that

$$
x^{q}=x^{q}(x g(x))=x^{q}(x g(x))^{2}=\cdots=x^{q}(x g(x))^{q} .
$$


Let $e=(x g(x))^{q}$. It is readily verified that $e^{2}=e$, and thus by (24), the lemma is proved.

The following three lemmas are well known and are stated without proofs.

LEMMA 8. If $[x, y]$ commutes with $x$, then for all positive integers $k$,

$$
\left[x^{k}, y\right]=k x^{k-1}[x, y]
$$

LEMMA 9. Let $R$ be a subdirectly irreducible ring. Then the only central idempotent elements of $R$ are 0 and 1 (if $1 \in R$ ).

LEMMA 10. Let $R$ be a weakly periodic ring. Then the Jacobson radical $J$ of $R$ is $\operatorname{nil}(J \subseteq N)$.

This lemma was proved in [2].

We are now in a position to prove our main theorems.

THEOREM 11. Let $R$ be a subweakly periodic ring such that the following two conditions hold:

(i) for all $x, y$ in $R$, there exist words $w(x, y)$ and $w^{\prime}(x, y)$ depending on $x$ and $y$ such that $w(x, y)[x y, y x]=0=[x y, y x] w^{\prime}(x, y)$;

(ii) the Jacobson radical $J$ is commutative.

Then $R$ is commutative. (In particular, this theorem holds if $R$ is weakly periodic (or periodic).)

Proof. By Lemma 7, we have for any $x$ in $R$,

$$
x \in J \quad \text { or } \quad x^{q}=x^{q} e, \quad e^{2}=e, q \geq 1 .
$$

As is well known, the ground $\operatorname{ring} R$ can be written as

$$
R \cong \text { a subdirect sum of subdirectly irreducible rings } R_{i} \quad(i \in \Gamma) .
$$

Let $\sigma_{i}: R \rightarrow R_{i}$ be the natural homomorphism of $R$ onto $R_{i}$. Let $x_{i} \in R_{i}$, and suppose that $x \in R$ such that $\sigma_{i}(x)=x_{i}$. By (26), we see that

$$
x_{i} \in \sigma_{i}(J) \quad \text { or } \quad x_{i}^{q}=x_{i}^{q} e_{i} \text { with } e_{i}=\sigma_{i}(e), e^{2}=e \in R, q \geq 1 .
$$

Moreover, by the proof of Lemma 7, we see that we can take $e=(x g(x))^{q}$ for some polynomial $g(\lambda) \in \mathbb{Z}[\lambda]$. Also, by Lemma 6 , $e$ is a central idempotent of $R$, and hence

$$
e_{i}=\left(x_{i} g\left(x_{i}\right)\right)^{q} \text { is a central idempotent of } R_{i} .
$$

We now distinguish two cases.

CASE 1. The ring $R_{i}$ does not have an identity. Suppose that there is an $x_{i} \notin \sigma_{i}(J)$. Then by (28), (29), and Lemma $9, x_{i}^{q}=0$ for some $q \geq 1$. Thus, $x_{i}$ is nilpotent, and hence by Lemma $5, x_{i} \in \sigma_{i}(J)$. This contradiction shows that 
$x_{i} \in \sigma_{i}(J)$ for all $x_{i} \in R_{i}$. By hypothesis, $J$ is commutative, and consequently, $R_{i}=\sigma_{i}(J)$ is commutative as well.

CASE 2. The ring $R_{i}$ has an identity 1 . Let $x_{i} \notin \sigma_{i}(J)$. By (28) and (29), $x_{i}{ }^{q}=$ $x_{i}^{q} e_{i}$, where $e_{i}=\left(x_{i} g\left(x_{i}\right)\right)^{q}$ is a central idempotent of $R_{i}$. Now, by Lemma 9 , $e_{i}=0$ or $e_{i}=1$. If $e_{i}=1$, then $\left(x_{i} g\left(x_{i}\right)\right)^{q}=1$, which implies that $x_{i}$ is a unit of $R_{i}$. On the other hand, if $e_{i}=0$, then $x_{i}^{q}=0$, and hence, by Lemma 5 , $x_{i} \in \sigma_{i}(J)$. The net result is

$$
\forall x_{i} \in R_{i}, x_{i} \in \sigma_{i}(J) \text { or } x_{i} \text { is a unit in } R_{i}
$$

Next, we claim that the set $N_{i}$ of nilpotents of $R_{i}$ is an ideal of $R_{i}$. To prove this, first recall that by Lemma $5, N_{i}$ is a commutative set, and hence $N_{i}$ is closed with respect to subtraction. Now, suppose that $a_{i} \in N_{i}, x_{i} \in R_{i}$. By Lemma 5, $a_{i} \in \sigma_{i}(J)$, and therefore, $a_{i}=\sigma_{i}(j)$ for some $j \in J$. Suppose that $x \in R$ is such that $x_{i}=\sigma_{i}(x)$. Then $a_{i} x_{i}=\sigma_{i}(j) \sigma_{i}(x)=\sigma(j x) \in \sigma_{i}(J)$ since $j \in J$. Since $J$ is commutative, $\sigma_{i}(J)$ is commutative, and hence

$$
\left[a_{i} x_{i}, a_{i}\right]=0 \quad\left(a_{i} \in N_{i}, x_{i} \in R_{i}\right)
$$

An easy induction shows that (31) implies that $\left(a_{i} x_{i}\right)^{q}=a_{i}{ }^{q} x_{i}^{q}$ for all positive integers $q$, and hence $a_{i} x_{i}$ is nilpotent. Similarly, $x_{i} a_{i} \in N_{i}$, and thus

$$
N_{i} \text { is a commutative ideal of } R_{i} \text {. }
$$

Our next goal is to show that

$$
\left[\left[a_{i}, u_{i}\right], u_{i}\right]=0 \text { for all units } u_{i} \text { in } R_{i} \text { and all } a_{i} \in \sigma_{i}(J) \text {. }
$$

To prove this, suppose that $a_{i}=\sigma_{i}(j)$ for some $j \in J$. Then $j$ is quasiregular, and hence $\sigma_{i}(j)$ is quasiregular as well, that is, $a_{i}$ is quasiregular. Therefore, $u_{i}{ }^{\prime}=1+a_{i}$ is a unit in $R_{i}$. By hypothesis (i), there exists a word $w\left(u_{i}, u_{i}{ }^{\prime}\right)$ such that $w\left(u_{i}, u_{i}{ }^{\prime}\right)\left[u_{i} u_{i}{ }^{\prime}, u_{i}{ }^{\prime} u_{i}\right]=0$. Since both $u_{i}$ and $u_{i}{ }^{\prime}$ are units in $R_{i}$, $w\left(u_{i}, u_{i}{ }^{\prime}\right)$ must also be a unit, and hence $\left[u_{i} u_{i}{ }^{\prime}, u_{i}{ }^{\prime} u_{i}\right]=0$, which is equivalent to

$$
\left[u_{i}\left(1+a_{i}\right),\left(1+a_{i}\right) u_{i}\right]=0 .
$$

Note that $\sigma_{i}(J)$ is a commutative ideal of $R_{i}$, and hence $\left[u_{i} a_{i}, a_{i} u_{i}\right]=0$. So, (34) implies that $\left[u_{i}, a_{i} u_{i}\right]+\left[u_{i} a_{i}, u_{i}\right]=0$, which is equivalent to $\left[u_{i},\left[a_{i}, u_{i}\right]\right]=$ 0 . This proves (33). Next, we prove that

$$
\sigma_{i}(J) \text { is contained in the center of } R_{i} \text {. }
$$


Suppose not. Then

$$
\left[a_{i}, b_{i}\right] \neq 0 \text { for some } a_{i} \in \sigma_{i}(J), b_{i} \in R_{i} .
$$

By (30), $b_{i} \in \sigma_{i}(J)$ or $b_{i}$ is a unit of $R_{i}$. As $\sigma_{i}(J)$ is commutative, (36) implies that $b_{i} \notin \sigma_{i}(J)$. Therefore,

$$
b_{i} \text { is a unit of } R_{i} \text {. }
$$

Moreover, by (36), we cannot have both $\left[a_{i}, 2 b_{i}\right]=0$ and $\left[a_{i}, 3 b_{i}\right]=0$. We assume, without loss of generality, that $\left[a_{i}, 2 b_{i}\right] \neq 0$. Letting $2 b_{i}$ play the role of $b_{i}$ in the argument which led to (37), we see that

$$
2 b_{i} \text { is a unit of } R_{i} \text {. }
$$

Let $b \in R$ such that $\sigma_{i}(b)=b_{i}$. By Lemma $4, b \in J$ or $b-b^{n} \in N$ for some integer $n>1$. Since $b_{i} \notin \sigma_{i}(J), b \notin J$, and hence

$$
b-b^{n} \in N \text { for some integer } n>1 \text {. }
$$

Applying a similar argument to $2 b_{i}$ yields

$$
2 b-(2 b)^{m} \in N \text { for some integer } m>1 .
$$

Now, by (39) and (40),

$$
b_{i}-b_{i}{ }^{n} \in N_{i}, \quad 2 b_{i}-\left(2 b_{i}\right)^{m} \in N_{i} .
$$

For any $x_{i} \in R_{i}$, let $\bar{x}_{i}=x_{i}+N_{i} \in R_{i} / N_{i}$. Then (41) implies that

$$
\left(\bar{b}_{i}\right)^{n}=\bar{b}_{i}, \quad\left(2 \bar{b}_{i}\right)^{m}=2 \bar{b}_{i}, \quad(n>1, m>1) .
$$

Observe that by (42),

$$
\begin{gathered}
\left(2 \overline{\boldsymbol{b}}_{i}\right)^{(m-1)(n-1)+1}=\left(2 \overline{\boldsymbol{b}}_{i}\right)^{m}=2 \overline{\boldsymbol{b}}_{i}, \\
\left(2 \overline{\boldsymbol{b}}_{i}\right)^{(m-1)(n-1)+1}=2^{(m-1)(n-1)+1}\left(\overline{\boldsymbol{b}}_{i}\right)^{n}=2^{(m-1)(n-1)+1} \overline{\boldsymbol{b}}_{i} .
\end{gathered}
$$

Hence,

$$
\left(2^{(m-1)(n-1)+1}-2\right) \bar{b}_{i}=\overline{0}
$$

Also, by (37), $\bar{b}_{i}$ is a unit of $R_{i} / N_{i}$, and hence the above equation implies that

$$
\left(2^{(m-1)(n-1)+1}-2\right) \overline{1}=\overline{0} .
$$


Therefore, $\left(2^{(m-1)(n-1)+1}-2\right) \cdot 1 \in R_{i}$ is nilpotent, and hence $R_{i}$ is not of characteristic zero. Since $R_{i}$ is subdirectly irreducible, we have

$$
\text { characteristic of } R_{i} \text { is } p^{k}, \quad p \text { prime, } k \geq 1 \text {. }
$$

Now, using (37), (42), and (46), we see that the subring $\left\langle\bar{b}_{i}\right\rangle$ generated by the unit $\bar{b}_{i}$ is a finite commutative ring with identity which has no nonzero nilpotents, and hence

$$
\left\langle\bar{b}_{i}\right\rangle \cong \bigoplus_{j=1}^{t} \mathrm{GF}\left(p^{k_{j}}\right), \quad t \text { finite, each } k_{j} \geq 1
$$

Let $\alpha=k_{1} k_{2} \cdots k_{t}$, then by $(47),\left(\bar{b}_{i}\right)^{p^{k \alpha}}=\bar{b}_{i}$. Thus

$$
b_{i}^{p^{k \alpha}}-b_{i} \in N_{i} \subseteq \sigma_{i}(J)
$$

Now, since $a_{i} \in \sigma_{i}(J)$ and $\sigma_{i}(J)$ is commutative, (48) yields $\left[b_{i}{ }^{k \alpha}-b_{i}, a_{i}\right]=0$. Thus

$$
\left[b_{i}^{p^{k \alpha}}, a_{i}\right]=\left[b_{i}, a_{i}\right]
$$

Combining (37), (33), Lemma 8, and (46), we see that (49) implies that $\left[b_{i}, a_{i}\right]=$ 0 , which contradicts (36). This contradiction proves (35). To complete the proof of the theorem, let $x_{i} \in R_{i}$ and let $x \in R$ be such that $\sigma_{i}(x)=x_{i}$. By Lemma 3, $N$ is an ideal of $R$, and therefore $N \subseteq J$. Now, by Lemma $4, x \in J$, in which case, $x-x^{n} \in J$ for any integer $n$ or $x-x^{n} \in N \subseteq J$ for some integer $n>1$, and hence, by (35), $x_{i}-x_{i}{ }^{n} \in \sigma_{i}(J) \subseteq$ Center of $R_{i}$. Thus, by a well-known Herstein [3, Theorem 21], $R_{i}$ is commutative. Therefore, $R$ is commutative, and this proves the theorem.

THEOREM 12. Let $R$ be a subweakly periodic ring such that the following two conditions hold:

(i) for all $x$ and $y$ in $R$, there exist relatively prime positive integers $m=$ $m(x, y)$ and $n=n(x, y)$ such that

$$
(x y)^{m}-(y x)^{m} \in C, \quad(x y)^{n}-(y x)^{n} \in C,
$$

where $C$ is the center of $R$;

(ii) the Jacobson radical $J$ is commutative.

Then $R$ is commutative. (In particular, this theorem holds if $R$ is weakly periodic (or periodic).)

Proof. Since $m$ and $n$ are relatively prime integers, there exist positive integers $k$ and $l$ such that $k m-l n=1$. Let $\gamma=\ln$. Then $k m=\gamma+1$. Moreover, 
by (50),

$$
\left[x y,(y x)^{m}\right]=0, \quad\left[x y,(y x)^{n}\right]=0,
$$

which implies

$$
\left[x y,(y x)^{k m}\right]=0, \quad\left[x y,(y x)^{l n}\right]=0 .
$$

Thus

$$
\left[x y,(y x)^{y+1}\right]=0, \quad\left[x y,(y x)^{\gamma}\right]=0 .
$$

Hence

$$
(y x)^{y+1} x y=x y(y x)^{y+1}=x y(y x)^{\gamma}(y x)=(y x)^{\gamma}(x y)(y x),
$$

and hence

$$
(y x)^{y+1} x y-(y x)^{y}(x y)(y x)=0
$$

Thus

$$
(y x)^{\gamma}\{y x \cdot x y-x y \cdot y x\}=0
$$

and hence

$$
(y x)^{\gamma}[x y, y x]=0 .
$$

A similar argument shows that $[x y, y x](y x)^{\gamma}=0$, and so

$$
(y x)^{\gamma}[x y, y x]=0=[x y, y x](y x)^{\gamma} .
$$

Theorem 12 now follows from (58) and Theorem 11 as well.

THEOREM 13. Let $R$ be a weakly periodic ring and suppose that the following conditions hold:

(i) for all $x, y$ in $R$, there exist words $w(x, y)$ and $w^{\prime}(x, y)$ such that $(x, y)[x y, y x]=0=[x y, y x] w^{\prime}(x, y)$

(ii) every commutator $[a, b]$, with $a$ and $b$ nilpotent, is potent $\left(i . e .,[a, b]^{k}=\right.$ $[a, b]$ for some integer $k>1$ ).

Then $R$ is commutative. (In particular, this theorem holds if $R$ is periodic.)

Proof. In view of Theorem 11, it suffices to show that $J$ is commutative. By Lemma 2 , the commutator idea $\mathscr{C}(R) \subseteq J$. Also, by Lemma $10, J \subseteq N$. Hence, $\mathscr{C}(R) \subseteq N$, and thus $N$ is an ideal of $R$. This implies that $N \subseteq J$, and hence $J=N$. Suppose that $a$ and $b$ are any elements of $N$. Then, $[a, b]$ is also in $N$ since $N$ is an ideal. Also, $[a, b]$ is potent by hypothesis (ii), and hence $[a, b]=0$ 
(since the only element which is both potent and nilpotent is zero). Therefore, $N=J$ is commutative. The theorem now follows from Theorem 11 .

THEOREM 14. Let $R$ be a weakly periodic ring such that the following conditions hold:

(i) for all $x, y$ in $R$, there exist relatively prime positive integers $m, n$ such that $(x y)^{n}-(y x)^{n} \in C$ and $(x y)^{m}-(y x)^{m} \in C$;

(ii) for all $a, b$ in $N,[a, b]$ is potent.

Then $R$ is commutative. (In particular, this theorem holds if $R$ is periodic.)

Proof. A careful examination of the proof of Theorem 12 shows that hypothesis (i) above implies that the "word" hypothesis stated in condition (i) of Theorem 13 holds here as well (see (58)). Thus, all of the hypotheses of Theorem 13 are satisfied, and hence $R$ is commutative.

We conclude by considering the special case where the "words" involved in the above theorems happen to be the empty words. As an illustration, we consider the status of Theorem 11 when $w(x, y)$ and $w^{\prime}(x, y)$ are the empty words. The result is the following corollary.

COROLlARY 15. Let $R$ be a subweakly periodic ring (in particular, $R$ may be chosen to be weakly periodic (or periodic)) satisfying the following conditions:

(i) $[x y, y x]=0$ for all $x, y$ in $R$;

(ii) $J$ is commutative.

Then $R$ is commutative.

A similar corollary may be obtained by taking the "words" in Theorem 13 to be the empty words.

Finally, we have the following corollary of Corollary 15.

COROLlARY 16. Let $R$ be a subweakly periodic ring (in particular, $R$ may be chosen to be weakly periodic or (periodic)) satisfying the following conditions:

(i) $[x, y]$ commutes with $x$ for all $x$ in $R$;

(ii) $J$ is commutative.

Then $R$ is commutative.

Proof. Let $x, y \in R$. By hypothesis (i) (interchanging $x$ and $y$ ), it follows that $[y, x]$ commutes with $y$, and hence $[x, y]$ commutes with $y$. Thus, $[x, y]$ commutes with both $x$ and $y$, and hence with $x y$. Thus, $y x$ commutes with $x y$, and Corollary 16 now follows from Corollary 15 .

\section{REFERENCES}

[1] H. E. Bell, On some commutativity theorems of Herstein, Arch. Math. (Basel) 24 (1973), 34-38.

[2] J. Grosen, H. Tominaga, and A. Yaqub, On weakly periodic rings, periodic rings and commutativity theorems, Math. J. Okayama Univ. 32 (1990), 77-81. 
[3] I. N. Herstein, A generalization of a theorem of Jacobson. III, Amer. J. Math. 75 (1953), 105-111.

[4] N. Jacobson, Structure of Rings, American Mathematical Society Colloquium Publications, vol. 37, American Mathematical Society, Rhode Island, 1964.

Amber Rosin: Department of Mathematics, University of California, Santa Barbara, CA 93106, USA

E-mail address: rosin@math.ucsb.edu

Adil Yaqub: Department of Mathematics, University of California, Santa Barbara, CA 93106, USA

E-mail address: yaqub@math.ucsb.edu 


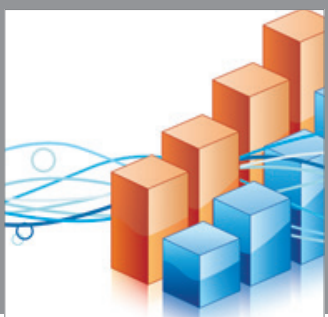

Advances in

Operations Research

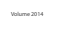

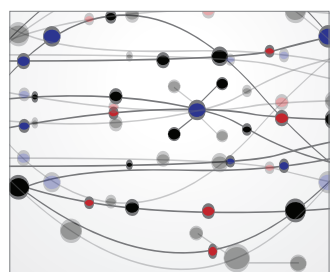

\section{The Scientific} World Journal
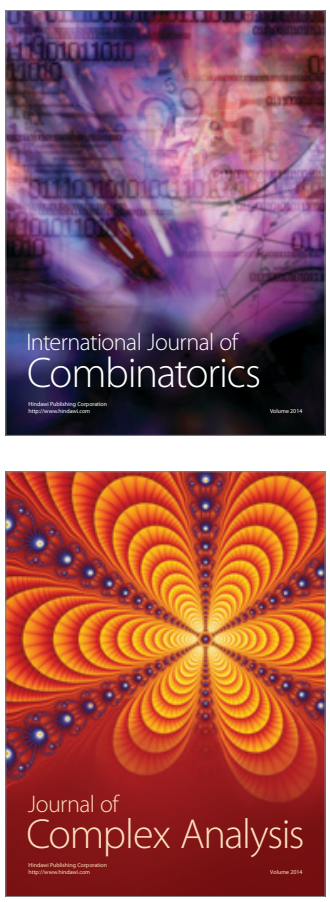

International Journal of

Mathematics and

Mathematical

Sciences
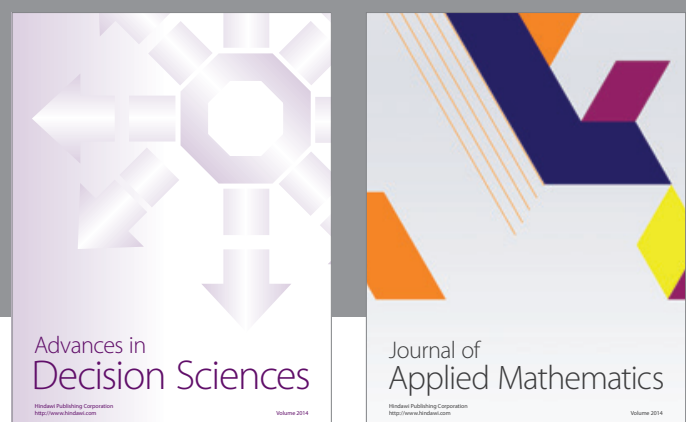

Journal of

Applied Mathematics
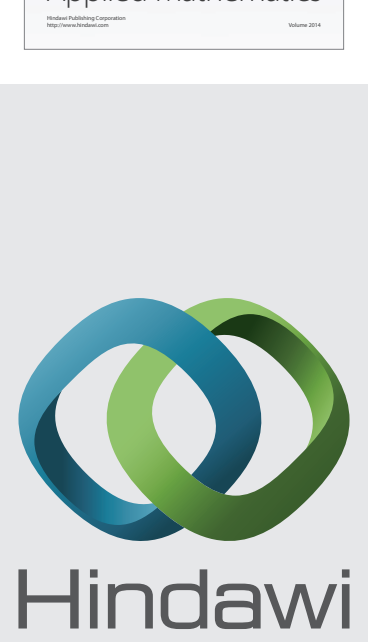

Submit your manuscripts at http://www.hindawi.com
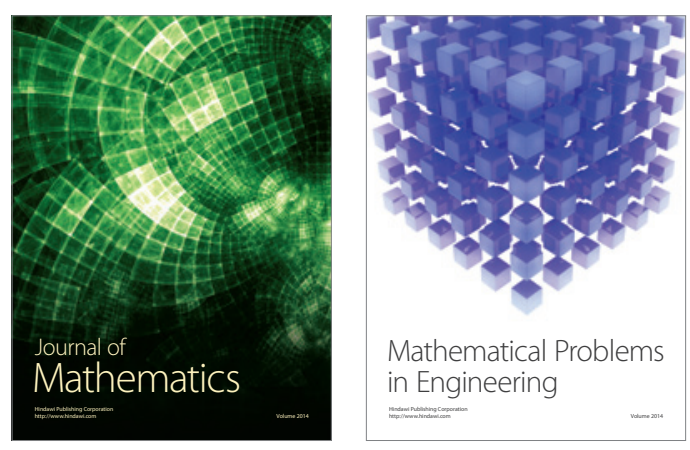

Mathematical Problems in Engineering
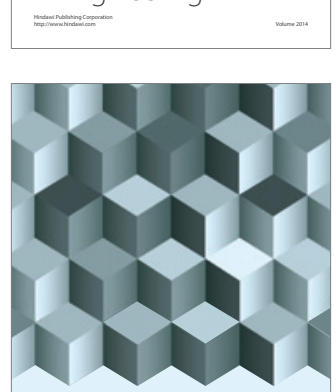

Journal of

Function Spaces
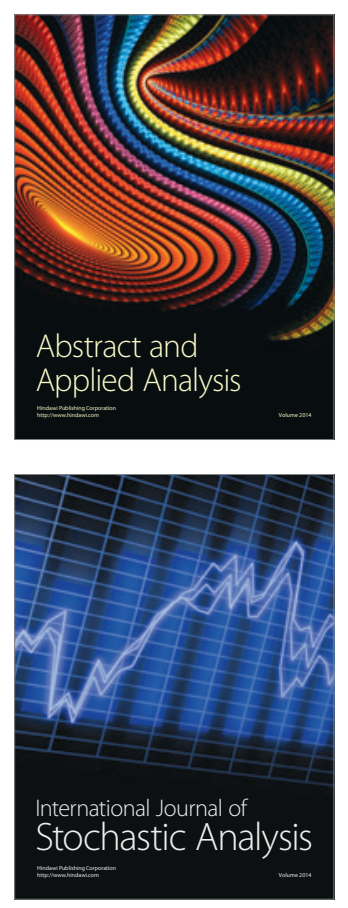

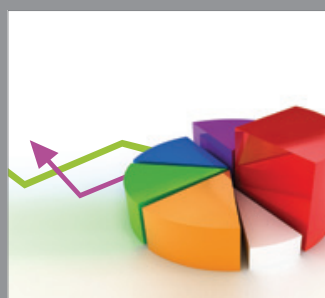

ournal of

Probability and Statistics

Promensencen
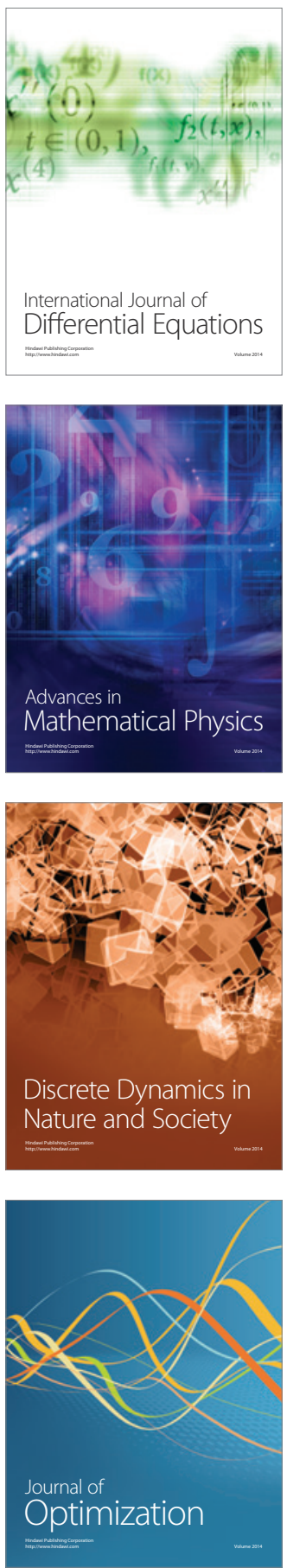